\title{
Les Petites Italies en France de 1945 à nos jours, des territoires singuliers
}

Little Italy in France from 1945 to the Present Day, Unique Territories La Piccola Italia in Francia dal 1945 a oggi, un territorio unico

\section{Aline Dumain}

\section{OpenEdition}

\section{Journals}

Édition électronique

URL : https://journals.openedition.org/cei/10569

DOI : 10.4000/cei.10569

ISSN : 2260-779X

Éditeur

UGA Éditions/Université Grenoble Alpes

Édition imprimée

ISBN : 978-2-37747-342-7

ISSN : 1770-9571

\section{Référence électronique}

Aline Dumain, "Les Petites Italies en France de 1945 à nos jours, des territoires singuliers », Cahiers d'études italiennes [En ligne], 34 | 2022, mis en ligne le 03 mars 2022, consulté le 04 mars 2022. URL http://journals.openedition.org/cei/10569; DOI : https://doi.org/10.4000/cei.10569

Ce document a été généré automatiquement le 4 mars 2022.

(c) ELLUG 


\title{
Les Petites Italies en France de 1945 à nos jours, des territoires singuliers
}

\author{
Little Italy in France from 1945 to the Present Day, Unique Territories \\ La Piccola Italia in Francia dal 1945 a oggi, un territorio unico
}

Aline Dumain

\section{Introduction}

$1 \mathrm{Si}$, en France, les grandes vagues migratoires italiennes se sont interrompues avec la fin des Trente Glorieuses, il reste néanmoins de très nombreuses traces bien vivantes de cette migration qui circulent dans la société française et qui s'enracinent dans les «Petites Italies ». Cette dénomination originellement appliquée à la situation nordaméricaine (Little Italy) «commence par une concentration italienne numériquement importante, exclusive ou dominante, avec à la clé, un marquage fort de l'espace et une organisation communautaire ${ }^{1} »$. Puis les années ont passé, les générations se sont succédé et les Petites Italies se sont transformées au gré des changements sociétaux. S'y développe ainsi un rapport aux territoires, aux frontières, à l'exercice des pouvoirs de l'État français mais aussi italien tout à fait singulier. Dans quelles mesures l'existence de ces territoires à forte empreinte italienne peut-elle s'articuler à celle d'un État centralisateur et à l'action publique ?

2 Les travaux de recherches mettent à jour deux types d'espaces qui peuvent être qualifiés de «Petite Italie »: des quartiers dans des villes comme Argenteuil, Grenoble, Nice ou Nogent-sur-Marne, et des espaces plus vastes dans lesquels la présence italienne est plus diffuse : Drôme, Franche-Comté, Nord, Normandie, Vosges. Par-delà la diversité qui caractérise les Petites Italies en France - dont l'étendue territoriale n'est qu'un aspect - elles se distinguent par certaines similitudes et notamment par une homogénéité culturelle que l'on qualifiera d'italianité. Comment circule-t-elle dans ces espaces? Quel rôle joue-t-elle, par-delà les différences, dans la permanence des Petites Italies? 
3 Pour répondre à ces interrogations, on analysera l'évolution de ces espaces qui échappent au découpage administratif étatique et qui sont faits d'un subtil équilibre entre tradition et modernité, garant de leur pérennité. On s'intéressera ensuite à l'italianité comme ciment identitaire, culturel mais aussi social de ces territoires alors qu'à partir des années 1950 circule dans la société française une italophilie de plus en plus marquée. Enfin on abordera le soutien de cette ethnicité par les institutions françaises et italiennes à travers les jumelages, l'enseignement de l'italien aux descendants d'immigrés. La situation de Marseille, étudiée notamment par Céline Regnard et Stéphane Mourlane ${ }^{2}$ sera analysée plus finement par une mise en perspective avec les Petites Italies en Lorraine du fer ${ }^{3}$.

\section{Propos liminaires}

Les Petites Italies, dans des approches comparatives, ont d'ores et déjà été beaucoup étudiées. Rien qu'en France, la littérature a vu la parution d'un ouvrage collectif consacré aux Petites Italies dans le monde ${ }^{4}$, un autre aux Petites Italies dans l'Europe du Nord-Ouest ${ }^{5}$. En revanche, pour la métropole proprement dite, si les ouvrages

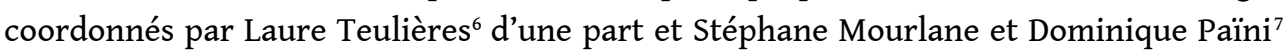
d'autre part présentent les lieux de l'italianité, l'emploi du terme "Petite Italie » est fait avec parcimonie. Il faut dire que les lieux à forte immigration italienne ne répondent pas toujours aux caractéristiques de la Petite Italie: "la concentration spatiale, le lien communautaire, l'ancrage urbain, l'homogénéité culturelle, etc. ${ }^{8}$ ». Deux des critères évoqués peuvent en effet être questionnés. C'est le cas pour la concentration spatiale car un certain nombre de régions en France ont connu une immigration italienne notoire mais leur présence reste plus diffuse : il en est ainsi de la Drôme, de la Franche-Comté, de la Normandie ou encore des Vosges.

Tableau 1. - Les Petites Italies en France. Recensement.

\begin{tabular}{|l|l|}
\hline Villes & Chercheurs ayant travaillé sur ce lieu \\
\hline Nogent & $\begin{array}{l}\text { Marie-Claude Blanc-Chaléard } \\
\text { Pierre Milza }\end{array}$ \\
\hline Nice & $\begin{array}{l}\text { Yvan Gastaut } \\
\text { Stéphane Mourlane }\end{array}$ \\
\hline Marseille « Petit Naples » & $\begin{array}{l}\text { Laure Teulières } \\
\text { Céline Regnard } \\
\text { Stéphane Mourlane }\end{array}$ \\
\hline Grenoble & Éric Vial \\
\hline Faubourg Saint-Antoine & Marie-Claude Blanc-Chaléard \\
\hline Argenteuil & Antonio Canovi \\
\hline Longwy-Villerupt & Claire Villaume-Canfora \\
\hline
\end{tabular}




\begin{tabular}{|l|l|}
\hline Sète & Laure Teulières \\
\hline Roubaix & Anna Soldado-Moine \\
\hline Dunkerque & Rudy Damiani \\
\hline Régions & \begin{tabular}{l} 
Frédéric Spagnoli \\
\hline «La mosaïque des Italiens » de Franche-Comte
\end{tabular} \\
\hline Vosges & Olivier Guatelli \\
\hline Drôme & Jean-Luc Huard \\
\hline Normandie & $\begin{array}{l}\text { Mariella Colin } \\
\text { Marc Pottier }\end{array}$ \\
\hline Nord & $\begin{array}{l}\text { Anna Soldado-Moine } \\
\text { Rudy Damiani }\end{array}$ \\
\hline Lot-et-Garonne & $\begin{array}{l}\text { Magalie Ceroni } \\
\text { Laure Teulières }\end{array}$ \\
\hline Lorraine & Marie-Louise Antenucci \\
\hline
\end{tabular}

5 Le second critère est celui de l'ancrage urbain : en effet, dans le Sud-Ouest, l'historienne Laure Teulières a bien décrit la forte présence italienne dans les campagnes ${ }^{9}$. Dans cet article, les Petites Italies seront considérées dans une acception extensive : l'ancrage local et urbain, s'il est fondamental car il rend unique chaque Petite Italie, est articulé à un transnationalisme qui dépasse les frontières de la cité. L'existence d'un lien communautaire et d'une homogénéité culturelle assure la pérennité des Petites Italies au fil des décennies.

6 En outre, illustrer son propos en donnant l'exemple de deux Petites Italies si différentes, Marseille et la Petite Italie de Lorraine $d u$ fer $^{10}$, peut sembler étonnant. En effet, on a d'une part, Marseille, grande ville cosmopolite, carrefour ancien de circulations et d'influences en raison de sa situation géographique portuaire ouverte sur le monde. Et d'autre part, les petites communes initialement rurales de Lorraine, telles que Jœuf ou Villerupt : leur développement important fut permis par la forte industrialisation de la fin du xix siècle, liée essentiellement aux mines de fer et aux usines sidérurgiques ${ }^{11}$.

7 La présence des Italiens à Marseille est fort ancienne ${ }^{12}$ tandis que la grande immigration transalpine date des années 1820 et :

À la veille de la guerre de 1914, la population italienne à Marseille oscille autour de 100000 personnes, ce qui équivaut à près du quart de la population totale de la ville; et elle représente plus des $4 / 5$ de la population étrangère résidant dans la cité phocéenne ${ }^{13}$. 
En Lorraine, cette immigration est plus tardive, elle date de la fin du XIX ${ }^{\mathrm{e}}$ siècle ; la première présence étrangère pour pallier le manque de main-d'œuvre dans l'extraction $\mathrm{du}$ fer est frontalière, belge et luxembourgeoise.

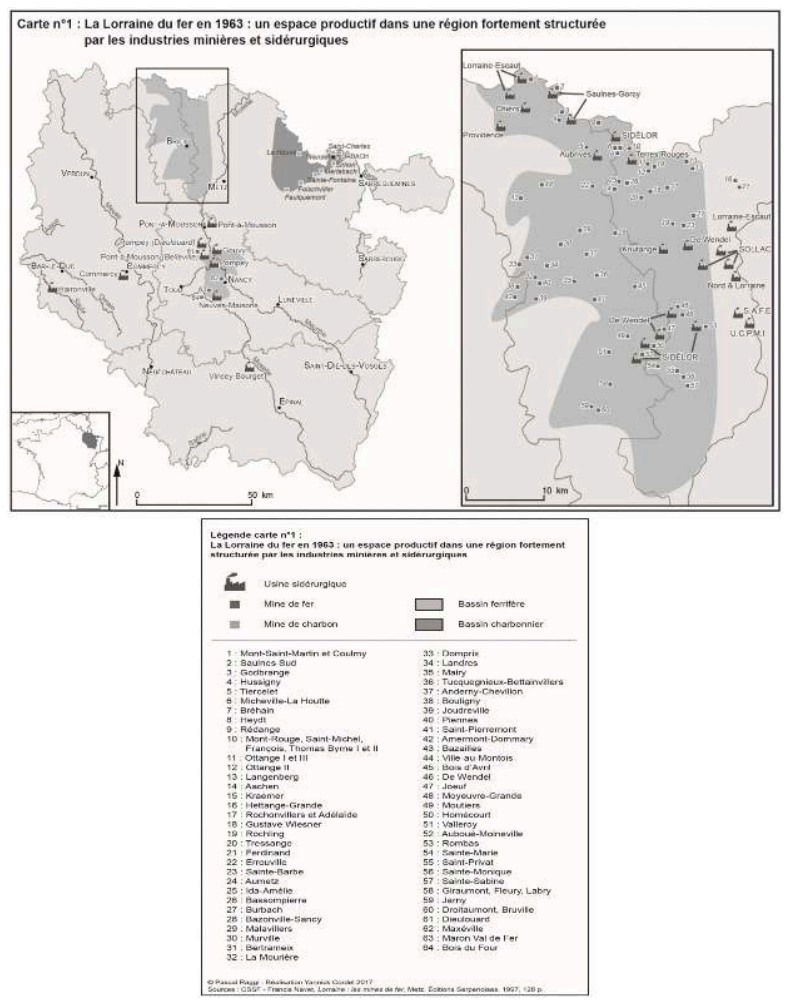

9 À la mono-industrie lorraine s'oppose également la diversité des emplois offerts par Marseille. Ce sont toutefois des emplois majoritairement industriels liés aux fonctions portuaires de la ville ou plus globalement :

Des secteurs d'activité réclamant l'emploi d'une main-d'œuvre nombreuse, peu qualifiée et à bas coût. [...] par exemple ses industries chimiques, métallurgiques, ses mines, ses carrières ; mais aussi le secteur du bâtiment et des travaux publics, le processus d'industrialisation se traduisant par la construction d'infrastructures modernes - canaux, routes, voies de chemin de fer - et par une forte croissance urbaine $^{14}$.

10 Or, par-delà les différences, les Petites Italies de Marseille et des cités lorraines présentent bien des similitudes, que l'on s'attachera à présenter dans cet article.

\section{Les Petites Italies en France, des territoires hétérogènes et singuliers}

11 Les Petites Italies en France se distinguent par de nombreuses caractéristiques des Petites Italies d'autres pays; c'est le cas des États-Unis ${ }^{15}$, mais pas seulement. La politique nationale et locale, le contexte culturel et social impriment une spécificité sur ces territoires qui, eux-mêmes, se différencient les uns des autres. 


\subsection{Des espaces atypiques dans un pays centralisé}

12 Le géographe Thomas Lacroix part d'un constat en apparence simple : « La territorialité juridique des États ne se manifeste pas de la même façon que la territorialité pratique des groupes sociaux ${ }^{16}$. » Les Petites Italies l'illustrent fort bien : ce sont des espaces dont le fonctionnement, les logiques échappent au découpage administratif de l'État français. L'entité territoriale qu'est le quartier n'est bien souvent pas pertinente, soit parce que les lieux de résidence sont liés au type d'emplois occupés, c'est le cas de Marseille; soit parce que l'importance de la communauté italienne suppose des habitations sur plusieurs quartiers.

Tableau 2. - Répartition des Italiens à Marseille.

\begin{tabular}{|l|l|l|}
\hline Localisation dans la ville & $\begin{array}{l}\text { Origines régionales des } \\
\text { Italiens }\end{array}$ & Type d'emplois \\
\hline $\begin{array}{l}\text { Vieille ville : Hôtel de Ville, Saint- } \\
\text { Jean, Saint-Laurent, Major }\end{array}$ & $\begin{array}{l}\text { Méridionaux, en particulier } \\
\text { de la région de Naples }\end{array}$ & $\begin{array}{l}\text { Navigateurs, pêcheurs, } \\
\text { ouvriers du port }\end{array}$ \\
\hline Le Lazaret, Arenc & & Ouvriers d'usines \\
\hline Saint-André, Saint-Henri, Estaque & Piémontais, Toscans & $\begin{array}{l}\text { Ouvriers dans les tuileries et } \\
\text { les industries chimiques }\end{array}$ \\
\hline $\begin{array}{l}\text { Saint-Louis, Belle-de-Mai, Saint- } \\
\text { Mauront }\end{array}$ & Piémontais, Toscans & $\begin{array}{l}\text { Ouvriers dans les raffineries, } \\
\text { huileries et stéarineries }\end{array}$ \\
\hline Menpenti, la Capelette & & Ouvriers dans les savonneries \\
\hline $\begin{array}{l}\text { Goudes, Bonneveine, Vieille } \\
\text { Chapelle, Montredon }\end{array}$ & & Journaliers agricoles \\
\hline Chartreux, Quatre Chemins & &
\end{tabular}

Tableau établi à partir des données recueillies dans l'ouvrage de Stéphane Mourlane et Céline Régnard, Empreintes italiennes. Marseille et sa région, Lyon, Lieux Dits, 2013, p. 26-27.

De plus, la formation des Petites Italies est à l'origine bien plus guidée par les lieux de travail et les patrons que par les politiques migratoires publiques. À Jœuf mais aussi dans toute la Lorraine du fer, les « barons du fer ${ }^{17}$ » ont exercé un paternalisme fort, qui " atteint un haut niveau de fonctionnement » dans l'après Seconde Guerre mondiale ${ }^{18}$, on y constate une emprise sur la vie des ouvriers, à la fois protectrice, codifiée (hiérarchisée) et autoritaire. De surcroît, si l'on s'attache à observer les premiers temps de la migration italienne, et donc la naissance d'une Petite Italie, on se rend compte qu'elle est fortement influencée par les régions voire les villages de départ. La diversité des Petites Italies s'explique en partie par la diversité des origines des Italiens. Pour Antonio Bechelloni,

Le poids des réalités locales de départ dont les dynamiques, sociales, culturelles, autant qu'économiques sont très diverses les unes des autres et s'inscrivent dans des chronologies spécifiques. [...] Elles semblent commander les destinations migratoires, la fréquence et l'importance des retours, le rythme suivi dans le 
relâchement des liens avec la communauté d'origine ou, inversement, le retour plus

ou moins définitif au sein de celle-ci ${ }^{19}$.

\subsection{Les Petites Italies, entre tradition et modernité}

16 Toutefois, ce sont les territoires d'accueil qui créent les Petites Italies, car au fil des dynamiques qui s'opèrent sur le territoire investi, une italianité, née en émigration, circule et transcende les appartenances régionales d'origine. Le temps et l'enracinement, souvent symbolisé par une demande d'accès à la nationalité française, font leur œuvre de modelage d'un territoire singulier. La Petite Italie peut être définie comme « à la fois conservatoire d'une culture rattachant le migrant à son pays natal et base à partir de laquelle va s'opérer la progressive intégration à son pays d'accueil ${ }^{23}$ ". Or, si cette définition s'applique à la Petite Italie dans ses débuts, cette dualité entre ethnicité et intégration, entre tradition et modernité pourrait-on dire, se complexifie au fil du temps, ses frontières se brouillent. La Petite Italie n'est plus seulement un entre-deux, mais un espace à part entière: "On saisit alors la construction d'une italianité, qui n'a pas beaucoup à voir avec la culture italienne, mais qui apparaît comme le produit de l'histoire de l'émigration - immigration et des réseaux ${ }^{24}$. Par bien des aspects, on peut se demander si les descendants d'Italiens, pour autant qu'ils se reconnaissent dans cette identité, ne sont pas des citoyens de la Petite Italie, plus que de la France ou de l'Italie. Ses ressortissants développent parfois un rapport à la tradition atypique, qui leur est propre et sur laquelle le temps semble s'être suspendu :

La venue d'Italiens dans la région, à l'occasion d'échanges scolaires par exemple, laisse toujours un peu rêveur l'observateur, qui constate que l'Italie "conservée " en Lorraine a fait du « sur place » et garde des habitudes que les Italiens d'Italie ont depuis longtemps rangés au rayon du folklore - chansons, associations, fêtes, etc. Fossilisation d'un passé constamment revisité où certains cultivent leurs origines comme on cultive son engagement passé ${ }^{25}$.

17 Les Italiens restés en Italie, lorsqu'ils viennent en Lorraine, font ainsi le constat d'une italianité que l'on pourrait qualifier de "désuète »: les pratiques sont parfois restées telles qu'elles l'étaient au moment de la migration. Toutefois, la Petite Italie, sous peine 
de se scléroser voire de disparaitre doit se renouveler. L'évolution des générations y contribue de manière naturelle pour ne pas dire inévitable. Les traditions ne sont pas tout à fait identiques; elles s'ancrent en effet dans une mémoire, dont l'oubli, facteur d'innovation, est une composante. Or on sait grâce à Maurice Halbwachs que: «La mémoire s'inscrit dans une temporalité singulière. La mémoire permet de vivre au présent et d'établir des ponts pour le futur. Elle est de ce fait toujours reconstruction du passée ${ }^{26}$. " La femme au foyer italienne est souvent présentée comme la gardienne de tradition ${ }^{27}$, qu'elle souhaite garder intactes. Or, elle les perpétue en les transformant. En cela, l'opposition entre modernité et tradition semble tout à fait inappropriée. Dans le cas des Siciliens de Farébersviller (Moselle Est) qui ont ressenti le besoin de manifester leur attachement à la Sicile lors de leur stabilisation en France, on peut parler de véritable « construction culturelle»:

Puisque nous sommes très attachés à la famille, à la mère, aux enfants, les premières fêtes qu'on a organisées, c'était l'Épiphanie (la fête des enfants) et la Fête des Mères disent les informateurs. Il est vrai que ces fêtes n'ont jamais fait partie de la tradition sicilienne et qu'elles ont été empruntées aux représentations de la société moderne, surtout en ce qui concerne la deuxième, mais elles ont été réinterprétées suivant les représentations traditionnelles ${ }^{28}$.

Enfin, les émigrés italiens impulsent une certaine modernité en participant à l'évolution de l'Italie et à son unification, notamment linguistique :

Si les Cavour, Mazzini, Garibaldi, Victor Emmanuel II ont « fait l'Italie », ce sont les millions d'émigrés qui, laissant le pays au moment de son unification politique ont contribué de façon particulière et souvent ignorée, "à faire les Italiens ", et ont porté dans le monde les valeurs et traditions qu'ils ont mises en relation (non sans conflits) avec les divers styles de vie des pays d'arrivée; ils ont créé de nouvelles identités et appartenances, souvent binationales. Qu'ils soient vénitiens, lombards, napolitains ou siciliens, ces émigrés se sont découverts, en émigration, surtout comme des «Italiens ", capables de redessiner de nouveaux liens avec le pays et la région d'origine ${ }^{29}$.

Nous reviendrons un peu plus tard sur la nature des liens entretenus avec le pays d'origine. Mais on mesure l'importance de l'émigré italien, qui ne se réduit pas à sa plus ou moins bonne intégration dans la société française. François Baudin attribue par ailleurs en partie aux Italiens l'unification de l'espace de la Lorraine du fer avant même le retour de la Lorraine annexée à la France en $1919^{30}$.

\subsection{Les différents temps des Petites Italies}

Pour chacune des Petites Italies, on peut constater une même dynamique de création, pérennisation et recomposition (parfois disparition). Seules les périodes changent en fonction de l'ancienneté du phénomène migratoire. Au démarrage, il y a une migration, essentiellement masculine souvent faite d'allers-retours entre la France et l'Italie. La migration devient ensuite plus familiale, notamment à partir des années 1920 avec l'arrivée des exilés antifascistes ${ }^{31}$. Les Petites Italies se structurent alors autour de lieux nouveaux : le logement en cité ouvrière se substitue progressivement à la cantina ${ }^{32}$; les commerces de produits italiens commencent à occuper l'espace urbain tandis que se tisse un réseau de sociabilités - associations italiennes, sportives et politiques avant la Seconde Guerre mondiale, plus souvent culturelles après 1945 - et d'entraides (Società di mutuo soccorso). 
21 Malgré l'avènement d'une "société de loisirs » notamment grâce à la baisse du temps de travail, la vie de ces Petites Italies se structure autour du travail. Très vite, elle repose également sur l'engagement syndical et politique. En Lorraine du fer, celui-ci s'attache à la lutte pour de meilleures conditions de travail et de salaires puis pour la survie de la mine et de l'usine. Il en va de même pour les autres Petites Italies, car partout le nombre d'emplois industriels diminuent tandis que les enfants des derniers Italiens arrivés accèdent à une promotion sociale : passés par l'école française, leurs parents les ont souvent poussés à ne pas exercer le même métier qu'eux. Gérard Mauger parle de génération ouvrière « désouvrierisée ${ }^{33}$ ». La désindustrialisation ${ }^{34}$ met à mal des identités basées sur l'appartenance au monde ouvrier faite à la fois de solidarités fortes liées à des métiers difficiles et sur une valorisation du mineur, de l'ouvrier sidérurgiste dans la société française ${ }^{35}$. L'engagement politique de gauche est aussi une caractéristique commune. Dans l'entre-deux-guerres, Loris Castellani observe que :

Peu de militants s'attelaient au travail politique parmi les immigrés. Car parmi les milliers, les dizaines de milliers d'hommes et de femmes qui prirent le chemin de l'exil afin d'échapper aux arrestations, de se soustraire aux persécutions du régime fasciste, peu continuaient la lutte à partir de l'étranger, beaucoup étaient démoralisés baissant définitivement les bras pour ne plus s'occuper que de gagner leur vie. [...] D'autre part, les tracasseries quotidiennes de la vie de l'émigré, les difficultés de la langue, la crainte de la perte du travail, la répression policière contribuaient à rendre inactifs de nombreux militants ${ }^{36}$.

Néanmoins, communisme et antifascisme marquent durablement les Petites Italies marseillaise et des cités du fer. Outre des raisons économiques, ce sont également pour ces motifs que les émigrés ont quitté l'Italie. Cet engagement s'illustre dans l'accession de descendants d'immigrés italiens à des fonctions municipales, conseillers voire maire. C'est le cas d'Henri Tasso dès 1935 à Marseille, le «plus célèbre des Italo-marseillais de la "deuxième génération" 37 ", d'Armand Sacconi, maire de Villerupt de 1959 à 1986 ou encore d'André Corzani, actuel maire de Jœuf.

Les Petites Italies ont-elles résisté à la promotion sociale, à la mobilité résidentielle (vers des zones pavillonnaires notamment), à la raréfaction des lieux communautaires tels que les commerces ou les cafés et aux retours en Italie? Ce sont en effet autant de facteurs susceptibles d'affaiblir le lien communautaire. Or, au-delà de l'absence ${ }^{38}$, à première vue, de toute trace d'italianité dans le paysage visuel, sonore mais aussi olfactif, on peut s'apercevoir çà et là de la vigueur de la culture italienne portée par les descendants d'immigrés. Vincent Ferry déclare en effet que : «Les signes d'italianité sont si visibles qu'ils sont devenus invisibles ${ }^{39}$.»

\section{L'italianité comme ciment identitaire, culturel et social des territoires}

Comment comprendre la permanence des Petites Italies en dépit de l'intégration de leurs ressortissants? Car, malgré ou peut-être justement grâce à leur invisibilité, les liens communautaires continuent de s'exprimer. C'est en effet une communauté qui a pu devenir invisible : sa couleur de peau ou sa religion majoritaire ne la renvoient pas systématiquement à son origine étrangère, comme cela peut être le cas des Africains ou des Juifs par exemple. Différents critères expliquent la permanence, pour ne pas dire la persistance des Petites Italies. Elles ont pu, nous l'avons vu, s'enraciner dans le 
territoire parce qu'elles étaient animées par des idéaux (sociaux, politiques...) autres que l'ethnicité, même si le partage de moments culturels et festifs ritualise le quotidien tout en exacerbant de manière positive l'appartenance identitaire. Alors que les piliers laborieux, syndicaux voire politiques ont partout décliné, l'italianité s'est montrée un puissant rempart contre la disparition des Petites Italies, en étant un ciment identitaire à la fois culturel et social des territoires de ces Petites Italies. Elle repose tout autant sur le regard de l'Autre, c'est-à-dire de la société française et des autres étrangers que sur la perception par les Italiens et leurs descendants d'eux-mêmes.

\subsection{Xénophobie et italophilie dans la société française depuis les années 1950}

Les deux mouvements inverses que sont la xénophobie et l'italophilie ont tous deux contribués à installer puis à maintenir les Petites Italies. La xénophobie dont ont été victimes les Italiens a eu pour corollaire de souder des liens de solidarité entre Transalpins et accentuer le rôle de sas, de refuge de la Petite Italie. Après 1945, les faits xénophobes à l'encontre des Italiens ont certes été d'une ampleur moins dramatique qu'à la fin du XIx ${ }^{e}$ siècle dans le sud de la France: "Vêpres marseillaises " ou le massacre d'Aigues-Mortes ${ }^{40}$, et au début du $\mathrm{xx}^{\mathrm{e}}$ siècle en Lorraine, théâtre de véritables "chasses à l'ours ${ }^{41}$ ». Néanmoins, elle reste une réalité, ponctuant le quotidien des Italiens les plus fraîchement arrivés comme parfois des Transalpins des premières vagues migratoires, souvent naturalisés. Les qualificatifs de « Macaroni », de « Rital » et tout un vocabulaire où transparaît un racisme que l'on qualifiera abusivement d'« ordinaire ${ }^{42}$ ", des stéréotypes et des préjugés fort tenaces, qui ressurgissent çà et là, encore aujourd'hui. Par ailleurs, après 1945, la migration transalpine concerne très majoritairement des Italiens du Sud. Dans la péninsule, la distinction est forte entre le Nord, industrialisé, qui s'enrichit et le Sud, très agricole et marqué par une grande pauvreté, dont beaucoup cherche à échapper en migrant justement vers le Nord ${ }^{43}$. Cette dichotomie se répercute sur les Petites Italies en France, notamment dans l'accueil réservé aux nouveaux arrivés. Dès 1932, Georges Mauco établit :

Une distinction très tranchée entre Italiens du Nord (un Nord, tout de même qui arrive jusqu'à la Toscane), censés être parfaitement " assimilables » et Italiens du Sud dont « des différences ethniques plus marquées, un rendement moindre dans le travail, une criminalité supérieure rendent l'assimilation plus complexe ${ }^{44} \ldots$

On peut trouver dans les archives les propos suivants :

La stabilité de cette main-d'œuvre italienne n'est donc pas possible tant qu'elle sera contaminée par cette recrudescence d'éléments calabrais et siciliens ; aussi serait-il désirable que des mesures fussent prises pour arrêter le recrutement de travailleurs immigrés dans le sud de l'Italie ${ }^{45}$.

Ceux-ci sont en effet jugés comme des "éléments troubles» qui influencent négativement les autres immigrés, venus du centre de l'Italie.

Or, l'italophilie circule aussi dans la société française à partir des années 1950. C'est d'ailleurs un phénomène mondial, l'importance des Italiens résidant hors de leur pays (oriundi) aux États-Unis, en Argentine et au Canada notamment y contribue très largement. L'Italie est la patrie des arts, du patrimoine architectural, pictural ${ }^{46}$, caractéristique exaltée et réactivée par le succès de son cinéma au lendemain de la Seconde Guerre mondiale. La Dolce Vita symbolise un art de vivre à l'italienne, aussi fascinant que scandalisant. À l'écran, la femme italienne rompt avec les stéréotypes, qui 
néanmoins perdurent dans des relations de voisinage. Cette richesse historique a contribué à transcender l'image négative habituelle de l'Italien, voleur du travail et de femmes. Les défauts des Italiens, à la fois réalités et stéréotypes, se muent en qualité. Claire Villaume dit en effet :

Toutes les habitudes de vie des Italiens étaient critiquées, comme par exemple la cuisine. Les journalistes s'en donnaient à cœur joie pour décrire avec dédain les plats italiens. De même, l'amour des Italiens pour la musique, leur façon d'exprimer leurs sentiments, leurs divertissements étaient dénigrés et méprisés. Bref, si aujourd'hui il apparaît normal d'affirmer que les cultures française et italienne sont similaires, à l'époque cela ne semblait pas aussi évident ${ }^{47}$.

$28 \mathrm{Au}$ lendemain de la Seconde Guerre mondiale, le regard dévalorisant au quotidien n'avait toutefois pas disparu: il faudra encore attendre plusieurs années avant que l'Italien cesse d'être le «Macaroni » ou le «Rital ", à l'école ${ }^{48}$ comme à l'usine : un mélange entre fascination et rejet entoure la présence des Italiens, alors que le racisme se tourne vers d'autres populations arrivées plus récemment, c'est le cas des Maghrébins.

La transformation du stéréotype, du négatif vers le positif, a contribué au maintien de ces territoires. Si la xénophobie a contribué à la fondation des Petites Italies, leur pérennisation repose, entre autres, sur l'italophilie. Dans ce contexte, on peut se demander si la Petite Italie n'est pas devenue également un « refuge » pour les Français, sans origine italienne? D'un point de vue positif, elle peut être vue comme le lieu d'une altérité joyeuse, acceptée et douce, le témoin d'une italophilie séculaire qui a passé outre les vicissitudes de périodes sombres ${ }^{49}$. D'un point de vue plus critique, la valorisation de la Petite Italie peut être vue comme une manière pour la société française de se donner "bonne conscience » dans sa façon de concevoir l'accueil des "populations venues d'ailleurs " ${ }^{50}$. Pierre Milza montre bien que l'idée selon laquelle les Italiens sont le modèle d'une intégration réussie cache les difficultés du passé tout en mettant à jour le fait que d'autres communautés ne parviennent pas à s'intégrer ${ }^{51}$.

\subsection{L'italianité dans les Petites Italies, une culture hybride}

Si la concentration dans les Petites Italies est un facteur favorable au maintien de l'italianité, on peut également dire que l'italianité peut aussi favoriser une unité territoriale. À Marseille comme dans les cités lorraines du fer, l'activité communautaire se tisse autour de lieux identiques, à quelques exceptions près : l'église, la Società di mutuo soccorso, les associations, le café et les commerces, le sport et les jeux. Les habitants des cités du fer doivent se rendre à Metz pour bénéficier des services du consulat et de la Società Dante Alighieri, contrairement aux Marseillais qui peuvent en profiter intra muros. En revanche, les logements en cités avaient souvent un jardin qui, outre une fonction vivrière importante pour les familles ouvrières ${ }^{52}$ avait également une fonction socialisatrice: avec les autres familles italiennes, mais aussi avec les mineurs et les sidérurgistes français ou polonais, également nombreux en Lorraine du fer. Au-delà de ces quelques différences, il existe une ambiance italienne ${ }^{53}:$ «[... il existe un entre-soi italien dans la région marseillaise, en grande partie informel, et donc moins aisément perceptible ${ }^{54}$.»

31 La Petite Italie ne continue d'exister que parce qu'elle est ouverte sur le monde et ses changements. La multiplicité des influences et des identités compose une culture hybride à ces descendants d'Italiens. Par exemple, les Italo-Lorrains de la jeune 
génération voient leur identité se construire entre des éléments des cultures italienne et française - elle-même traversée par l'italophilie, mondialisée, par la culture juvénile et enfin par la culture de la Lorraine du fer, à la fois friche industrielle, marge frontalière et territoire ouvert sur la construction européenne. Michel Dreyfus montre bien à travers le portrait d'Alberto Balducci, figure syndicale de la Lorraine du fer, la dualité voire la multiplicité des appartenances identitaires des Petites Italies ${ }^{55}$. Politiquement, le communisme qu'il pratique semble être propre aux Petites Italies du fer ${ }^{56}$. L'influence du Parti communiste italien y est forte :

[...] entre les années 1920 et les années 1970, la sphère politique et idéologique italienne a constitué un modèle pour des fractions des forces politiques et/ou pour des milieux culturels français. Ce fut le cas du fascisme à partir du début des années 1920 qui exerça un rayonnement international incontestable. [...] Après 1945, ce furent le pôle communiste et marxiste, et, dans une moindre mesure, le pôle socialiste-libéral rassemblé pour une courte période autour du parti d'Action (1942-1947) qui suscitèrent un vif intérêt dans certains milieux politico-culturels de gauche français. Le PCI et les cercles culturels membres ou proches du PCI étaient vus en France comme les dépositaires d'un communisme et d'un marxisme souples et ouverts, particulièrement sur les questions idéologiques et culturelles. Ces représentations tranchaient avec l'image d'un PCF inféodé à l'URSS, réceptacle d'un marxisme dogmatique, rigide sur les questions culturelles et ayant du mal à rassembler autour de lui l'ensemble de l'intelligentsia de gauche ${ }^{57}$.

Professionnellement, les mineurs de fer, en dépit de la proximité géographique, sont bien différents des mineurs de charbon ${ }^{58}$. Les luttes, qui auraient pu être communes en raison de motifs similaires (la fermeture des mines) ne l'ont été qu'à deux reprises : en 1963 et en 1968. En toile de fond, il y a une distinction entre la Lorraine allemande et la Lorraine française (de 1871 à 1919), qui persiste bien des années après. Même parmi les hommes du fer, le métier exercé les distingue : les destinées des mineurs de fer sont différentes de celles des sidérurgistes. Bernard Lahire montre bien que les «dissonances culturelles» des individus ${ }^{59}$ sont liées à des variations intraindividuelles : les mobilités sociales, scolaires ou professionnelles, les contraintes ou influences relationnelles (celle des pairs, des conjoints, des collègues de travail), la baisse d'intensité de la croyance en la culture littéraire et artistique. Comme tout individu, l'Italien d'origine se construit une culture hybride et multiple, une identité polymorphe. Le sociologue Marco Martiniello déclare en effet :

L'ethnicité n'est qu'une forme de classification sociale et politique et une forme d'inégalité structurelle parmi d'autres. En effet, les relations et les clivages ethniques sont étroitement liés à d'autres formes de relations et de clivages sociaux entre classes sociales, entre genres ou encore, entre États-nations ${ }^{60}$.

Les divergences sont multiples et ne se réduisent pas à l'environnement professionnel.

\subsection{Une culture transnationale}

Les descendants d'immigrés se caractérisent par la multiplicité des appartenances identitaires, développant ainsi un rapport au territoire qui leur est propre: le transnationalisme est un concept approprié pour penser l'itinéraire des descendants d'Italiens. Le transnationalisme peut être défini comme l'espace, imaginaire ou réel, créé par les migrants ou leurs descendants entre leur pays d'origine et leur pays d'accueil ; il est donc un déplacement des identités qui reposent habituellement sur le territoire national vers un territoire qui le transcende ${ }^{61}$. L'article de Tiziana Caponio et Camille Schmoll avance un certain nombre de caractéristiques qui définissent le 
transnationalisme. Ces éléments s'appliquent particulièrement bien à la situation des descendants d'Italiens et donc à la persistance de l'italianité dans les Petites Italies: «[...] les liens avec le pays d'origine - disent-elles - ne sont pas nécessairement voués à disparaître en une génération, mais, au contraire, ils peuvent se réactiver ou se reconstruire au fil du temps ${ }^{62}$. $»$ En Lorraine du fer, les entretiens avec des jeunes de la troisième génération ${ }^{63}$ (voire des générations suivantes) témoignent d'un intérêt pour la culture italienne et d'une volonté de maintenir ou de renouer avec ses racines. "Les attachements transnationaux - peut-on également lire dans cet article - ne se traduisent pas nécessairement en cultures d'opposition ou en comportements déviants ${ }^{64}$.» Cette culture se construit de manière "pacifique ", discrète et apaisée. On a vu l'importance prise dans la société française par l'italophilie, des particularités de cette communauté qui a su devenir invisible. "Le transnationalisme chez les enfants d'immigrés - expliquent-elles également - ne se traduit pas nécessairement par un engagement constant et régulier, mais par un "bricolage" d'actions différenciées sur les plans politique, culturel ou religieux réalisé de manière discontinue ${ }^{65} »$, par l'intrication des deux cultures. Ce transnationalisme, que l'on peut qualifier de culturel, évolue par exemple au gré des événements festifs, qui galvanisent le sentiment communautaire: le parcours de l'équipe d'Italie à la Coupe du monde ou à l'Euro de football ; à l'école, la participation au Festival du film italien de Villerupt (Meurthe-etMoselle) ou le voyage en Italie organisé par le professeur d'italien ajoutent une expérience sensible et sensorielle à la connaissance de la langue du pays.

\subsection{L'identité et les différentes générations}

On peut lire enfin dans cet article que :

Le transnationalisme, loin d'être une forme de reproduction des modes de vie et des appartenances des parents, est une réinvention, à partir de contextes et d'expériences divers. Par conséquent, les formes prises par le transnationalisme chez les enfants d'immigrés sont multiples, du repli face à la stigmatisation à l'utilisation stratégique des liens transnationaux comme ressource ${ }^{66}$.

On a, dans un paragraphe précédent, réfléchi à l'articulation subtile entre tradition et modernité, cette forme d'adaptabilité permanente à l'air du temps. La culture des ItaloFrançais est en effet toujours une réinvention. À la différence de leurs aînés pour qui l'Italie est le territoire quitté, c'est-à-dire regretté ou honni, l'Italie des descendants d'Italiens est beaucoup plus intellectualisée et nationale. Si le lien affectif n'a pas disparu chez les générations nées en France, il est d'une toute autre nature et s'articule à une appréhension plus globale de la culture italienne. À l'instar de l'italophilie mondialisée, leur culture transcende le localisme, sans toutefois le renier. Le voyage en Italie pendant les vacances n'a ainsi pas la même signification : les aînés retournent dans leur village pour passer du temps avec leur famille restée en Italie, les enfants y ajoutent un tourisme culturel ou de villégiature, faisant écho à la connaissance générale de la culture du pays que leur transmet l'école : « Les deuxièmes ou troisièmes générations expriment, de leur côté, une demande culturelle, englobant à la fois la culture savante italienne et la culture "folklorique" des parents et grands-parents ${ }^{67}$. »

Pour la plupart des migrants à leur arrivée, la langue de communication est le dialecte $^{68}$; la langue transmise par l'école aux petits émigrés qui souhaitent apprendre la langue d'origine est l'italien. Les variations et les niveaux de maitrise linguistiques sont très importants. 
Dans les récits autobiographiques des membres de la première génération, on retrouve beaucoup le sentiment de nostalgie. Nostalgie du pays quitté, mais aussi nostalgie de ce que fut la Petite Italie dans le passé. La Petite Italie en Lorraine du fer dans les années 1950 n'a en effet rien de commun avec celle de 2015. Pour illustrer les différences selon les générations, l'histoire du Festival du film italien de Villerupt est un exemple intéressant. En 1976, un groupe de jeunes passionnés de cinéma lance un festival de trois jours et tourne un film ${ }^{69}$ qui rend hommage au travail de leurs pères et se déroule à Villerupt, cité du fer alors en pleine reconversion industrielle. Face au succès de la première édition, le Festival est reconduit et prend de l'ampleur au fil des années. Le caractère "ethnique ${ }^{70}$ " se construit peu à peu autour de nombreux bénévoles qui cuisinent des spécialités italiennes : l'artisanat et le bénévolat donnent une valeur d'authenticité à cet événement qui entre dans la tradition de cette Petite Italie du fer. On y vient tout autant pour manger et parler italien que pour voir un film. En 2021, alors que se profile la $44^{e}$ édition, la nostalgie parcourt les spectateurs les plus anciens : nostalgie d'un pays "perdu », l'Italie et ses régions, qui s'incarne à l'écran, nostalgie des autres lieux disparus ou morcelés, la mine, l'usine, la Petite Italie, dont les traces ont parfois été effacées; et nostalgie enfin d'un événement devenu rituel, le festival, mais qui a perdu ses caractéristiques ethniques les plus marquantes : la cuisine des mammas italiennes a laissé la place à des traiteurs tandis que l'événement a pris une dimension nationale, attirant parfois un public venu de loin. À l'image des Petites Italies, le festival illustre bien les divergences générationnelles ${ }^{71}$ et le sentiment de nostalgie ${ }^{72}$.

Les Petites Italies ne sont pas dénuées de tensions - peut-être même de conflits générationnelles. Les processus d'intégration dans la société française, d'acculturation et le maintien d'éléments ethniques, propres à chaque individu et à chaque famille forgent des réalités polymorphes. Face à cette population, les États, français et italien, et les différentes institutions renforcent leurs rôles, se posant en promoteurs de ce qu'on pourrait nommer une " politique culturelle des Italiens de France».

\section{Le rôle des pouvoirs français et italien}

L'État français, à partir des années 1970, accompagne cet élan communautaire. Dans le même temps, le démarrage de la construction européenne à six fait de la France et de l'Italie des partenaires privilégiés. En outre, l'État italien, à travers son ambassade à Paris et ses consulats disséminés dans les grandes villes françaises, « prend soin » de ses ressortissants émigrés en France. La zone géographique de ces consulats s'est agrandie à mesure que certains ont fermé. C'est le cas par exemple des Consolati Generali d'Italia :

\begin{tabular}{|l|l|}
\hline De... & Fermé le... \\
\hline Bastia & 31 octobre 2007 \\
\hline Chambéry & 30 novembre 2008 \\
\hline Mulhouse & $1^{\text {er juillet } 2010}$ \\
\hline
\end{tabular}




\begin{tabular}{|l|l|}
\hline Lille & $1^{\mathrm{er}}$ juillet 2011 \\
\hline Toulouse & 27 novembre 2013 \\
\hline
\end{tabular}
maintenant un service de proximité aux ressortissants italiens. Il reste donc désormais cinq consulats : Paris, Lyon, Marseille, Nice et Metz. Le maillage territorial de l'espace français est donc moins important, ce qui correspond probablement à des besoins moindres tandis que la digitalisation des démarches administratives accentue cette réalité.

41 Toutes les actions initiées par les pouvoirs publics de part et d'autre des Alpes ont une portée diplomatique voire politique et contribuent au maintien des Petites Italies.

\subsection{Les jumelages}

42 À travers les jumelages mis en place au lendemain de la Seconde Guerre mondiale, la volonté politique des élus locaux est clairement de nouer des relations avec des villes étrangères là où les États-nations échouent sur le plan diplomatique en raison notamment des antagonismes de la guerre froide. Tout comme la Communauté économique européenne, les jumelages avaient pour but de rétablir une paix durable sur le continent européen. Cette coopération entre collectivités territoriales ${ }^{73} \mathrm{~s}^{\text {'est }}$ particulièrement bien développée dans les années $1950^{74}$; elle concerne alors essentiellement des villes européennes. À l'heure actuelle, l'Italie est le troisième pays européen avec lequel les villes françaises ont tissé des liens avec 1002 villes jumelées, derrière l'Allemagne (2317) et le Royaume-Uni (1093). Cette importance numérique de l'Italie comme de l'Allemagne ${ }^{75}$ témoigne du rôle politique de ces jumelages ${ }^{76}$.

Le jumelage avec des villes italiennes n'a pas pour objectif immédiat de satisfaire la communauté italienne présente sur la commune française. Toutefois, la carte des jumelages illustre que le choix des villes n'est pas dû au hasard. Le maire de la commune d'Audun-le-Tiche (Moselle) explique en effet le choix de la commune de Gualdo Tadino ${ }^{77}$ par « une population italienne très importante sur Audun-le-Tiche et sur tout le secteur minier et sidérurgique particulièrement de la région d'Ombrie et des Marches. Donc la région d'Ombrie ». Vient s'ajouter le rôle déterminant du maire de la commune qui « était Monsieur Angel Filipetti qui a toute sa famille à Gualdo Tadino, il est originaire de là-bas, c'était le papa de notre actuelle ministre ${ }^{78}$ ". L'histoire du jumelage de Moutiers (Meurthe-et-Moselle) avec Bagno di Romagna ${ }^{79}$ est similaire : un nombre important d'habitants originaires de ce même village articulé à l'impulsion de plusieurs personnalités marquantes de la commune, parmi lesquels l'artiste Amilcar Zannoni ${ }^{80}$. On doit toutefois se garder de considérer le lien entre émigration et jumelage en règle générale tant les contre-exemples sont nombreux ${ }^{81}$. Toutefois, parmi les 34 villes du département des Bouches-du-Rhône concernées par le jumelage avec l'Italie, la majorité (24) a signé des accords de coopération avec des villes du nord de l'Italie, officialisant un lien migratoire ancien. Marseille est jumelée avec Gênes et un projet culturel organisé en $2013^{82}$ dynamise une coopération qui dans bien des villes connaît un ralentissement ${ }^{83}$, tout en réactivant un patrimoine, une histoire commune, de villes méditerranéennes tournées vers le monde. Le jumelage des villes lorraines avec des communes italiennes n'a pas la même signification. Leur nombre est déjà plus 
réduit, ce qui peut s'expliquer par une distance géographique plus grande avec l'Italie que le département des Bouches-du-Rhône. On constate également une plus grande disparité des régions italiennes représentées. La forte immigration en provenance des régions du sud de l'Italie dans les années 1950-1960 explique probablement cette situation. Dans les années 2000, leurs dynamismes varient beaucoup selon les communes, selon la présence ou non d'un établissement scolaire qui participe à la coopération par des échanges linguistiques. À Audun-le-Tiche par exemple, le jumelage avec Gualdo Tadino implique les élèves italianisants du collège.

Si les jumelages ont perdu de leur importance dans les années 2000, ils ont toutefois permis aux Petites Italies de se pérenniser à une période où les liens avec l'Italie tendaient à s'amenuiser (années 1970-1980, fin des grandes vagues d'immigration transalpine, importance des naturalisations et enracinement de la seconde génération). Les Petites Italies ont, d'une certaine manière, bénéficié de la tension entre État et

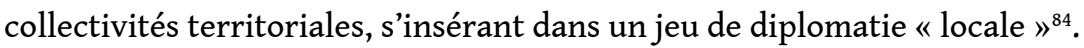

\subsection{L'enseignement de l'italien aux descendants d'immigrés}

La langue et sa transmission sont des éléments-clés dans la pérennisation de la Petite Italie. Elle est un support communicationnel, mais aussi un vecteur de culture: l'enseignement de l'italien ouvre les portes d'une histoire commune, qui dépasse le cercle familial. L'enseignant d'italien, outre un passeur linguistique, est un passeur de savoirs et de sens, c'est-à-dire de savoirs sensoriels. La langue donne accès à l'évolution du pays, à travers un dialogue possible avec la famille restée en Italie, la lecture de la presse, la télévision ou plus récemment Internet et les réseaux sociaux. Dans une certaine mesure, les Italiens de France - comme d'ailleurs - ont utilisé la langue italienne comme langue commune alors que majoritairement, dans la péninsule, on continuait d'utiliser le dialecte comme langue quotidienne, l'italien restant l'idiome de l'école et des institutions, de l'unification relativement récente de la péninsule (1861).

Les pouvoirs italien et français ont très vite perçu l'importance de la transmission de l'italien dans le maintien de l'italianité parmi les Transalpins. Il s'agissait de préparer le retour des petits émigrés dans leur pays d'origine. Mais, cet enseignement d'abord dispensé par des associations est empreint de considérations idéologiques : fascistes dans les années 1930, communistes dans les années 1950-19605. L'attitude des autorités françaises oscillent entre méfiance (années 1930) et contrôle rigoureux des activités des associations italiennes ${ }^{86}$.

47 L'institutionnalisation des cours d'italien prodigués aux enfants d'immigrés dans les années 1970 est le résultat de plusieurs éléments qui se combinent. La création des Enseignements en langue et culture d'origine ${ }^{87}$ vient entériner, dans le cas de l'italien, l'existence d'un enseignement déjà fort contrôlé par l'État et conforté par une directive européenne ${ }^{88}$. Du reste, dans les années 1970, s'engage en France une politique davantage tournée vers une prise en compte de l'interculturalité, alors que de nouvelles vagues migratoires et la décolonisation questionnent l'identité de la France et apportent un nouveau regard sur l'étranger. Cette politique n'est toutefois pas dénuée de critiques et d'interrogations. Abdelmalek Sayad déclare :

Donc, donner à ces élèves "particuliers " de l'école française «la possibilité de maintenir un contact avec leur langue et leur culture maternelles ", soit. Mais à proprement parler, est-ce là le rôle propre de l'école française ou, tout au moins, son rôle premier et direct ${ }^{89}$ ? 
Cette implication des politiques publiques dans la dynamisation d'une identité ethnique peut être considérée comme une implication dans la vie privée, familiale ou associative des individus; elle peut être vue comme un élément d'une politique culturelle, qui justement tient compte de la pluralité des cultures et cherche à permettre leur expression. Indéniablement, elle est enfin un pan dans le jeu de diplomatie culturelle avec l'Italie.

\section{Conclusion}

L'avènement puis le maintien au fil des décennies des Petites Italies a introduit au sein de l'espace français un mode d'appropriation du territoire et un rapport aux pouvoirs français et italien tout à fait singuliers. Le local s'articule au transnational alors que s'élabore dans la seconde moitié $\mathrm{du} \mathrm{xx}^{\mathrm{e}}$ siècle une conscience européenne. L'italianité qui circule dans ces espaces est faite d'éléments en apparence contradictoires - intégration et ethnicité, tradition et modernité, mémoire et oubli - dont on a pu observer la cohérence. Par leur position marginale, les Petites Italies offrent un poste intéressant pour observer les États français et italien dans leur fonctionnement et leur jeu de diplomatie culturelle.

Toutes les pistes de réflexion n'ont pas été explorées dans cet article, par définition synthétique. C'est notamment le cas de la question des générations d'Italiens de France, qui mériteraient une analyse plus approfondie; c'est le cas également de l'activité des associations culturelles, de l'implication des États dans leur financement, de leur articulation entre le local et le national; et enfin la place des médias dans la pérennisation de l'italianité et des Petites Italies.

\section{NOTES}

1. M.-C. Blanc-Chaléard, "Introduction», dans M.-C. Blanc-Chaléard, A. Bechelloni, B. Deschamps, M. Dreyfus et É. Vial (éds), Les Petites Italies dans le monde, Rennes, Presses universitaires de Rennes, 2007, p. 16.

2. S. Mourlane et C. Régnard, Empreintes italiennes. Marseille et sa région, Lyon, Lieux Dits, 2013.

3. La Lorraine du fer s'étend « de la frontière luxembourgeoise jusqu'au bassin de Nancy, et du Pays-Haut de Meurthe-et-Moselle jusqu'aux vallées usinières du Nord-Ouest mosellan » (P. Raggi, La désindustrialisation de la Lorraine du fer, Paris, Garnier, 2019, p. 20). L'unité de cet espace réside dans son histoire industrielle et migratoire.

4. Voir note 1 ci-dessus.

5. J. Rainhorn (éd.), Petites Italies dans l'Europe du Nord-Ouest. Appartenances territoriales et identités collectives à l'ère de la migration italienne de masse (milieu du XIX siècle - fin $d u \quad X^{e}$ siècle), Valenciennes, Presses universitaires de Valenciennes, 2005.

6. L. Teulières (éd.), Italiens 150 ans d'émigration en France et ailleurs, Toulouse, Editalie, 2013.

7. S. Mourlane et D. Païni, Ciao Italia! Un siècle d'immigration et de culture italiennes en France, Paris, La Martinière, Musée national de l'histoire de l'immigration, 2017. 
8. S. Dufoix et V. Foucher, « Les Petites Italies (et les autres...). Éléments de réflexion sur la notion d'ethno-territoire », dans M. Dreyfus et É. Vial (éds), Les Petites Italies dans le monde, ouvr. cité, p. 424.

9. L. Teulières (éd.), Immigrés d'Italie et paysans de France. 1920-1944, Toulouse, Presses universitaires du Mirail, 2002.

10. Étudiée notamment par l'historienne M.-L. Antenucci : L'immigration en Moselle (1870-1940): L'exemple des Italiens, thèse de doctorat en histoire contemporaine sous la direction de A. Wahl, soutenue à Metz en 2000 et Parcours d'Italie en Moselle : histoire des immigrations italiennes, 1870-1940, Metz, Éditions Serpenoise, 2005, 267 p. ; et P.-D. Galloro, Ouvriers du fer, princes du vent. Histoire des flux de main-d'œuvre dans la sidérurgie lorraine (1880-1939), Metz, Éditions Serpenoise, 2001.

11. La découverte en 1877 par deux scientifiques britanniques (Sidney Thomas et Percy Gilchrist) d'un procédé permettant l'extraction du fer à grande échelle a entraîné le développement industriel autour des mines de fer et des usines sidérurgiques; les besoins en main-d'œuvre y étaient conséquents.

12. J.-F. Dubost, La France italienne: $X V I^{e}-X V I I^{e}$ siècles, Paris, Aubier, 1997.

13. É. Temime, "Les Italiens dans la région marseillaise pendant l'entre-deux-guerres », dans P. Milza (éd.), Les Italiens en France de 1914 à 1940, Rome, École française de Rome, 1986, p. 547.

14. S. Mourlane et C. Régnard, Empreintes italiennes, ouvr. cité, p. 15.

15. J. Rainhorn, Paris, New York : des migrants italiens, années 1880-1930, Paris, CNRS éditions, 2005.

16. T. Lacroix, Le Transnationalisme : Espace, Temps, Politique, Paris, Université Paris-Est, 2018, p. 14.

17. J.-M. Moine, Les barons $d u$ fer. Les maîtres de forges en Lorraine du milieu du XIX ${ }^{e}$ siècle aux années trente. Histoire sociale d'un patronat sidérurgique, Nancy, Presses universitaires de Nancy, 1990.

18. V. Ferry, «Du rejet des Italiens à la culture italo-lorraine : un siècle pour une intégration riche mais difficile ", dans Italiens en Lorraine: de l'intégration à la réussite, Chambre de commerce italienne pour la France, 1997, p. 88-89.

19. A. Bechelloni, «L'Histoire de l'immigration-émigration italienne en perspective: France, Italie, Amérique », Cahiers des Annales de Normandie, $\mathrm{n}^{\circ}$ 28, 1998, p. 73.

20. F. Sirna, «Une émigration italienne ou des émigrations d'Italie?», dans L. Teulières (éd.), Italiens, 150 ans, ouvr. cité, p. 49.

21. S. Mourlane et C. Régnard, Empreintes italiennes, ouvr. cité, p. 18.

22. P. Milza et M.-C. Blanc-Chaléard, Le Nogent des Italiens, Paris, Autrement, 1995, p. 27.

23. M.-C. Blanc-Chaléard, Les Italiens dans l'Est parisien. Une histoire d'intégration (1880-1960), Rome, École française de Rome, 2000, p. 11.

24. G. Campani, «L'exemple de la diaspora italienne ", Social Science Information, vol. $31, \mathrm{n}^{\circ} 2$, 1992, p. 341.

25. L. Delmas, R. Martinois et S. Sutera-Sardo, " "Petites Italies" en Lorraine sidérurgique au début du $\mathrm{xx}^{\mathrm{e}}$ siècle, dans J. Rainhorn (éd.), Petites Italies dans l'Europe du Nord-Ouest, ouvr. cité, p. 97.

26. M. Halbwachs, Les cadres sociaux de la mémoire, Paris, Albin Michel, 1994 (1925).

27. Son rapport à l'intégration et à l'ethnicité est plus complexe qu'il ne l'apparaît de prime abord.

28. S. Palidda, «Famiglia siciliana di Farebersviller e Dintorni », La Trace, $n^{\circ}$ 2-3, p. 55-56.

29. A. Bechelloni, «L'Histoire de l'immigration-émigration », Cahier des Annales de Normandie, art. cité.

30. F. Baudin, Histoire économique et sociale de la Lorraine, t. 3 : Les hommes, 1870-1914, Metz, Éditions Serpenoise, 1997.

31. G. Noiriel, «Les immigrés italiens en Lorraine pendant l'entre-deux-guerres: du rejet xénophobe aux stratégies d'intégration », dans P. Milza (éd.), Les Italiens en France de 1914 à 1940, ouvr. cité, p. 609-632. 
32. L. Prencipe, Les émigrations italiennes, 1840-2008, Conférence « Le temps des Italiens : approches, cultures et territoires» (20 octobre 2009, Marseille), <https://archives.approches.fr/IMG/File/ Les_emigrations_italiennes.pdf>, p. 4.

33. G. Mauger, Âges et générations, Paris, La Découverte, 2015, p. 56-57.

34. Les mines de fer ferment dans la seconde moitié $d u x^{e}$ siècle jusqu'à la dernière en 1997 à Audun-le-Tiche (Moselle). Voir P. Raggi, La désindustrialisation de la Lorraine du fer, Paris, Garnier, 2019.

35. P. Raggi, Le mineur de fer au travail, Metz, Éditions Serpenoise, 2007, p. 194-229.

36. L. Castellani, «Un aspect de l'émigration communiste italienne en France : les groupes de langue italienne au sein du PCF (1921-1928)», dans P. Milza (éd.), Les Italiens en France de 1914 à 1940, ouvr. cité, p. 201.

37. É. Temime, Les Italiens dans la région marseillaise, art. cité, p. 559-560.

38. P. Milza, Voyage en Ritalie, Paris, Payot, 2010, p.424. Cet historien utilise le terme de «transparence ».

39. V. Ferry, conférence à l'IUT de Longwy le 5 juin 1996 sur le thème «En passant par la Lorraine, j'ai vu l'Italie ».

40. E. Barnabà, Mort aux Italiens! 1893, le massacre d'Aigues-Mortes, Villefranche-sur-Rouergue, Éditalie Éditions, 2012 ; G. Noiriel, Le massacre des Italiens. Aigues-Mortes, 17 août 1893, Paris, Pluriel, 2018.

41. P.-L. Buzzi, "Affrontements xénophobes et identités : les "chasses à l'italien" en Lorraine (années 1890-1910)», Histoire@Politique, n 32, 2017.

42. Au sens de banal car banalisé.

43. «Entre 1955 et 1965, plus de 2 millions de personnes quittent les régions du Mezzogiorno pour celles du Centre-Nord. » (A. Delpirou, Atlas de l'Italie contemporaine, Paris, Autrement, 2011.)

44. G. Mauco, Les étrangers en France : leur rôle dans l'activité économique, Paris, Armand Colin, 1932, cité par A. Bechelloni, art. cité.

45. Archives départementales de Meurthe-et-Moselle, 1 W 1438, Activité de l'Office national de l'Immigration, signé par le sous-préfet.

46. En Allemagne, par exemple, Yvonne Rieker constate que : « Les immigrés italiens profitèrent, d'une certaine manière, du "regard touristique", de beaucoup d'Allemands sur l'Italie, regard qui commença à émerger dans les années 1950. Les principaux attributs pour l'Italie étaient le soleil et la mer, le chant et la musique, le vin et la gastronomie, l'amour et la joie de vivre, la beauté du paysage et le charme des villes anciennes. S'y ajoutait parfois la référence, typique de la bourgeoisie cultivée, à l'Italie comme berceau de la culture de l'Antiquité et de la Renaissance. » (Y. Rieker, "Un "bout d'Italie" en Allemagne? L'immigration italienne dans la Ruhr depuis 1955 ", dans M. Dreyfus et É. Vial (éds), Les Petites Italies dans le monde, ouvr. cité, p. 231.) 47. C. Villaume, Les Petites Italies, Metz, Édition Serpenoise, 2001, p. 104.

48. Louise Canette montre bien la dualité de l'accueil réservé aux enfants à l'école : «Les enfants d'immigrés italiens ayant fui Mussolini ont ainsi pu être bien accueillis par des instituteurs républicains partageant des opinions politiques parfois proches de celles de leurs parents, nombreux sont les témoignages et autobiographies regorgeant de souvenirs gratifiants des écoliers se disant remarquablement bien intégrés au sein de l'École républicaine. Cependant, d'autres entretiens entre les historiens et les enfants d'origine italienne, mettent en avant la sensation d'exclusion et d'humiliation ressentie par ces derniers sur les bancs des classes de l'Hexagone. » (L. Canette, Les enfants d'immigrés italiens dans les écoles françaises. Parcours scolaires de 1935 à 1955, Sarrebruck, Éditions universitaires européennes, 2012, p. 5-6.)

49. Laurent Dornel a montré dans son ouvrage La France hostile. Socio-histoire de la xénophobie l'importance de la xénophobie à partir des années 1870 et son articulation avec la construction de la République. 
50. V. Viet, Histoire des Français venus d'ailleurs de 1850 à nos jours, Paris, Perrin, coll. "Tempus », 2003.

51. A. Cordeiro, « Le paradoxe de l'immigration portugaise », Hommes et Migrations, $\mathrm{n}^{\circ} 1123,1989$, p. 25-32 ; cet auteur évoque « le paratonnerre maghrébin », c'est-à-dire que la montée du racisme anti-Maghrébins dans les années 1970 a contribué à " protéger la communauté portugaise » du racisme dont elle a pu être victime dans les années 1960 . On constate un phénomène similaire pour la communauté italienne.

52. "Chaque ménage ouvrier avait un potager, car les patrons voulaient donner aux ouvriers une occupation saine. » (C. Villaume, Les petites Italies, Metz, Éditions Serpenoise, 2001, p. 72.)

53. B. Bégout, Le concept d'ambiance, Paris, Seuil, 2020. Ce philosophe définit ainsi le concept d'ambiance : «C'est cette connaissance spontanée et irréfléchie qui, lors d'une veillée funèbre par exemple, permet à une personne arrivant à l'improviste de saisir l'ambiance de deuil et de recueillement qui règne, et d'ajuster ses expressions et son comportement à ce climat affectif. ». (Entretien accordé à Michel Eltchaninoff, rédacteur en chef de la revue Philosophie Magazine, publié le 21 octobre 2020.)

54. S. Mourlane et C. Régnard, Empreintes italiennes, ouvr. cité, p. 18.

55. «La trajectoire d'Alberto Balducci dans cette région minière et sidérurgiste de forte immigration italienne n'est pas non plus exempte d'une certaine marginalité au sein du Parti communiste, ce qui renforce encore un peu davantage l'hypothèse d'une Petite Italie syndicale dans le bassin de Trieux. [...] au sein de l'univers communiste lorrain, les mineurs sont constamment dans une situation marginale. » (M. Dreyfus, Petites Italies dans l'Europe du NordOuest, ouvr. cité, p. 186 et 188.)

56. P. Basse, L'italianité du Parti communiste dans le bassin sidérurgique de Longwy : de la reconstruction du pays à celle du parti, mémoire de maîtrise, Université Nancy 2, 1992.

57. O. Forlin, «Introduction. - La culture italienne en France au $x^{\mathrm{e}}$ siècle: circulation de modèles et transferts culturels. Approches historiographique et problématiques ", Cahiers d'études italiennes, $\mathrm{n}^{\circ}$ 28, 2019, <https://doi.org/10.4000/cei.5286>, § 12-13.

58. Les mines de fer sont situées à l'ouest du département de la Moselle, les mines de charbon à l'est.

59. B. Lahire, Laculture des individus. Dissonances culturelles et distinction de soi, Paris, La Découverte, 2004.

60. M. Martiniello, Penser l'ethnicité. Identité, culture et relations sociales, Liège, Presses universitaires de Liège, 2013, p. 32-33.

61. R. Fibbi et G. D'Amato, "Transnationalisme des migrants en Europe : une preuve par les faits ", Revue européenne des migrations internationales, vol. 24, $\mathrm{n}^{\circ} 2,2008, \mathrm{p} .7,<\mathrm{https} / /$ doi.org/ 10.4000/remi.4452>, § 1 .

62. T. Caponio et C. Schmoll, «Enfants d'immigrés et transnationalisme : une lecture des travaux italiens ", Migrations Société, vol. 24, n 141-142, 2012, p. 240, <https://www.cairn.info/revuemigrations-societe-2012-3-page-239.htm>, § 3.

63. Par exemple avec des jeunes résidant à Villerupt : Sébastien Ortoleva (entretien du $1^{\mathrm{er}}$ février 2014) et Arnaud Scalia (entretien du 14 février 2014).

64. T. Caponio et C. Schmoll, «Enfants d'immigrés et transnationalisme : une lecture des travaux italiens », Migrations Société, art. cité, § 23.

65. Ibid., §5.

66. Ibid., $\S 84$.

67. G. Campani, «L'exemple de la diaspora italienne », art. cité, p. 334.

68. En Moselle, en 1974, le dialecte est utilisé à $49 \%$ entre les conjoints de la première génération, ce pourcentage chute à 17,9 \% lorsque ceux-ci s'adressent à leurs enfants (Archives de Moselle, 74 J 26, citées par N. Lilti-Huguet, Scolarisation et formation professionnelle des enfants de 
travailleurs italiens et portugais en Moselle, Centre d'informations et d'études d'économie humaine en Lorraine, 1974, p. 57).

69. J.-P. Menichetti, L'anniversaire de Thomas, Villerupt, Studio 16, 1982.

70. J.-M. Leveratto, Cinéma, spaghettis, classe ouvrière et immigration, Paris, La Dispute, 2010.

71. Entretien avec Antoine Compagnone, délégué général du festival (20 janvier 2021).

72. Entretiens avec Grazio Di Blasi (3 février 2013), habitant de Villerupt et habitué du festival et Alain Barberi, un des membres de la première équipe du festival (20 janvier 2013).

73. Majoritairement des communes.

74. En 1940, seules cinq collectivités territoriales avaient signé des conventions de jumelage; en 1960 , le nombre de ces conventions était presque de 1000.

75. On peut lire à ce sujet la thèse qui sera prochainement soutenue par Constanze Knitter et qui est consacrée aux Jumelages entre la France et la République démocratique allemande (1959-1989).

76. C. Chombard-Gaudin, "Pour une histoire des villes et communes jumelées ", Vingtième Siècle. Revue d'histoire, $\mathrm{n}^{\circ} 35,1992$, p. 60-66.

77. Commune de la province de Pérouse dans la région Ombrie (Italie centrale).

78. Entretien avec Lucien Piovano, maire d'Audun-le-Tiche, 23 janvier 2014.

79. Commune de la province de Forlì-Cesena, en Émilie-Romagne (Italie du Nord).

80. Entretien avec Rémy Vidili, maire de Moutiers, 6 février 2014.

81. Jean-Luc de Ochandiano constate en effet que les jumelages de communes de l'agglomération lyonnaise avec des communes italiennes n'ont en général pas de lien avec l'histoire migratoire (J.-L. de Ochandiano, Lyon à l'italienne. Deux siècles de présence italienne dans l'agglomération lyonnaise, Lyon, Lieux Dits, 2013).

82. Il s'agit d'un programme européen Comenius Regio « Med Patrimoine » qui réunit les deux cités autour d'une même thématique : les embarcations traditionnelles de pêche, la barquette marseillaise et le gozzo génois. Ce programme regroupe plusieurs événements, parmi lesquels une conférence et la présentation de maquettes de bateaux réalisés par des lycéens marseillais et génois.

83. Si les années 1990 ont vu un renforcement des conventions de coopération, les années 2000 enregistrent un ralentissement de ces jumelages. En 1992, Cécile Chombard-Gaudin s'interrogeait déjà dans la conclusion de son article: "On peut se demander en outre si la disparition de la guerre froide jointe à la facilité actuelle des voyages ne retire pas un peu de leur sens aux jumelages. » (C. Chombard-Gaudin, art. cité, p. 66.)

84. La mise en place des jumelages en France a connu une histoire tumultueuse (C. ChombardGaudin, art. cité).

85. Archives départementales de Moselle, $11 \mathrm{~T} 109$ et $1431 \mathrm{~W} 35$.

86. C'est le cas par exemple de l'Association éducatrice franco-italienne (voir Archives départementales de Moselle, 106 W 12, Accord du 26 avril 1950 entre l'État et l'AEFI portant sur l'enseignement du français et de l'italien aux immigrés italiens et à leurs enfants).

87. Circulaire $\mathrm{n}^{\circ} 75-148$ du 9 avril 1975.

88. Directive du 25 juillet 1977.

89. A. Sayad, L'école et les enfants de l'immigration. Essais critiques, Paris, Seuil, 2014. 


\section{RÉSUMÉS}

La présence désormais ancienne d'immigrés italiens sur le territoire français a créé une mosaïque de Petites Italies. Dans ces espaces singuliers, cohabitent de nombreuses générations de descendants transalpins; une italianité aux influences multiples se mêle à un rapport transnational à l'Italie et à la France tandis que l'italophilie qui circule dans la société française depuis les années 1950 contribue à maintenir vivantes les spécificités culturelles de ces territoires. En outre, les Petites Italies échappent au découpage administratif de l'État français, fort centralisé. Ce dernier soutient toutefois cette ethnicité et contribue ainsi, lui aussi, au maintien des Petites Italies à travers les jumelages ou l'enseignement de l'italien aux descendants d'immigrés. Les Petites Italies deviennent un objet de diplomatie culturelle entre la France et l'État italien, acteur incontournable dans la pérennisation de la langue notamment.

The long-standing presence of Italian immigrants on French territory has created a mosaic of Petites Italies. In these singular spaces, many generations of transalpine descendants live together, an Italianness with multiple influences is mixed with a transnational relationship to Italy and France, while the Italophilia that has been circulating in French society since the 1950s contributes to keeping the cultural specificities of these territories alive. The Little Italies don't correspond to the administrative division of the highly centralised French state. However, the latter supports this ethnicity and thus also contributes to the maintenance of Little Italy through city twinnings and the teaching of Italian to descendants of immigrants. This is part of a cultural diplomacy between the French and the Italian state, a key player in the perpetuation of the language.

La lunga presenza di immigrati italiani sul territorio francese ha creato un mosaico di Petites Italies. In questi spazi singolari convivono molte generazioni di discendenti transalpini, un'italianità dalle molteplici influenze si mescola a un rapporto transnazionale con l'Italia e la Francia, mentre l'italofilia che circola nella società francese dagli anni'50 contribuisce a mantenere vive le specificità culturali di questi territori. Le Piccole Italie sfuggono alla divisione amministrative dello stato francese altamente centralizzato. Tuttavia, quest'ultimo sostiene questa etnicità e quindi contribuisce anche al mantenimento delle Little Italy attraverso i gemellaggi e l'insegnamento dell'italiano ai discendenti degli immigrati. Sostiene in tal modo una relazione di diplomazia culturale con lo stato italiano, attore chiave nella perpetuazione della lingua.

\section{INDEX}

Keywords : Italian identity, Italian language, generations, integration, Italianophilia, twinning, Little Italies, territory, tradition, transmission, transnationalism, xenophobia

Parole chiave : gemellaggio, generazioni, integrazione, italianità, italofilia, lingua italiana, Piccole Italie, territorio, tradizione, transnazionalità, trasmissione, xenofobia

Mots-clés : générations, intégration, italianité, italophilie, jumelages, langue italienne, Petites Italies, territoire, tradition, transmission, transnationalisme, xénophobie 
AUTEUR

ALINE DUMAIN

Laboratoire CRULH, Université de Lorraine

aline.dumain@univ-lorraine.fr 